\title{
Understanding 'Non-genetic' Inheritance: Insights from Molecular-Evolutionary Crosstalk
}

\author{
Irene Adrian-Kalchhauser (1D, , ${ }^{1, \star, @ ~ S o n i a ~ E . ~ S u l t a n, ~}{ }^{2}$ Lisa N.S. Shama (D), ${ }^{3}$ Helen Spence-Jones, ${ }^{4}$ \\ Stefano Tiso, ${ }^{5}$ Claudia Isabelle Keller Valsecchi $\mathbb{C}^{0},{ }^{5}$ and Franz J. Weissing ${ }^{6}$
}

\begin{abstract}
Understanding the evolutionary and ecological roles of 'non-genetic' inheritance (NGI) is daunting due to the complexity and diversity of epigenetic mechanisms. We draw on insights from molecular and evolutionary biology perspectives to identify three general features of 'non-genetic' inheritance systems: (i) they are functionally interdependent with, rather than separate from, DNA sequence; (ii) precise mechanisms vary phylogenetically and operationally; and (iii) epigenetic elements are probabilistic, interactive regulatory factors and not deterministic 'epialleles' with defined genomic locations and effects. We discuss each of these features and offer recommendations for future empirical and theoretical research that implements a unifying inherited gene regulation (IGR) approach to studies of 'non-genetic' inheritance.
\end{abstract}

\section{Inheritance beyond DNA Poses Key Questions for Evolution and Ecology}

Biologists are currently engaged in a lively conversation about whether it is necessary to expand our view of biological inheritance to include 'non-genetic' factors [1-3]. In particular, molecular epigenetic mechanisms (such as DNA methylation, histone modifications, and small noncoding RNAs) have been interpreted as additional 'streams' of phenotypic information distinct from DNA sequence transmission [4-8]. In comparison with DNA sequence variation, which is transmitted with great fidelity across numerous generations, these factors have complex and potentially unpredictable dynamics: they may arise stochastically or be induced by specific environmental conditions, and they may persist from one to several generations (reviewed in [9-16]). Researchers in many fields are now confronting an unexpected question: must we fundamentally revise our understanding of inheritance to incorporate these new insights?

For evolutionary biologists, the phenotypic impact of inherited 'non-genetic' factors and their potential contribution to adaptation and diversification are pressing issues. Although many questions remain, mounting evidence indicates that these transgenerational mechanisms may substantially influence phenotypic outcomes in a wide range of organisms (reviewed in [17-22]). Induced inherited effects may be negative: for instance, parent individuals with a nutrient-poor, high-fat, or high-sugar diet may transmit altered DNA methylation states to offspring that result in metabolic or developmental disorders [23-25]. Alternatively, stressful parental conditions may induce gametically transmitted changes that promote adaptive phenotypes in offspring encountering similar stresses [26-32]. Because (unlike allele frequency change) such induced, inherited effects could cause adaptive adjustments in many individuals in a population (and their descendants) after only one generation, they are of particular interest to ecologists and evolutionary biologists as a potential mechanism for rapid adaptation to environmental changes [33,34].

In addition to phenotypic effects on individual organisms, 'non-genetic' factors may influence the adaptive potential of populations [6,35-37]. Recent studies of wild populations have shown the

\section{Highlights}

'Non-genetic' inheritance (NGI) involves a wide range of epigenetic, cytoplasmic, and other mechanisms. The term inherited gene regulation (IGR) provides a unifying concept for the diverse heritable factors that may alter offspring gene expression.

It is crucial to be aware of three features of NGl systems: they are functionally dependent with, rather than separate from, DNA sequence information; the precise mechanisms are highly diverse within and across taxa; and they act probabilistically rather than as 'epialleles' with defined genomic locations and effects.

These often-overlooked properties point to promising empirical and theoretical research avenues in evolution and ecology. A group of molecular and evolutionary biologists offers an integrated perspective on this emergent research area.

${ }^{1}$ Centre for Fish and Wildlife Health, Department for Infectious Diseases and Pathobiology, Vetsuisse Faculty, University of Bern, Länggassstrasse 122, 3012 Bern, Switzerland

${ }^{2}$ Biology Department, Wesleyan University, Middletown, CT 06459, USA ${ }^{3}$ Coastal Ecology Section, Alfred Wegener Institute Helmholtz Centre for Polar and Marine Research, Wadden Sea Station Sylt, Hafenstrasse 43, 25992 List, Germany

${ }^{4}$ Centre for Biological Diversity, School of Biology, University of St Andrews, St. Andrews, UK

${ }^{5}$ Institute of Molecular Biology (IMB), Ackermannweg 4, 55128 Mainz, Germany 
role of epigenetic variation in several aspects of population dynamics relevant to local divergence [38-42], invasion potential [43,44], migration propensity [45], developmental morph determination [46,47], and host-parasite interactions [48-50]. Note that field studies are challenged to determine the precise source of epigenetic variation (i.e., direct environmental induction, inheritance from previously induced generations, or locally selected 'epialleles' [51]), and to exclude the possibility that epigenetic differences among populations are simply downstream consequences of genetic differences [52,53]. Theoretical models indicate that 'non-genetic' factors, despite their transient nature, may substantially change selection gradients and heritabilities, and hence alter evolutionary trajectories [7-9,54,55].

\section{Toward a More Precise View of 'Non-genetic' Inheritance}

Although much progress has been made in understanding the ecological and evolutionary role of 'non-genetic' inheritance (NGI) [37,52,56], further advances may be hampered by three common simplifications about these molecular systems (for mechanistic reviews [13,57-59]). First, the very term 'non-genetic' is inaccurate: inherited epigenetic factors and DNA sequence are not distinct but functionally interdependent [52,53]. Second, processes such as DNA methylation are not uniform mechanisms, but operate in a multiplicity of ways depending on both species and mode of induction (e.g., [60,61]). Third, most epigenetic variants are not deterministic 'epialleles' with defined genomic locations and effects, but probabilistic, interactive regulatory factors [62].

By translating a fine-grained literature on molecular mechanisms into broader properties, we provide a more precise basis for integrating 'non-genetic' modes of inheritance into evolutionary and ecological studies. The key shared features of these mechanisms can be clarified by the unifying concept of inherited gene regulation (IGR; Box 1). This term encapsulates the common effect of a wide range of transgenerational systems that can alter genome activity and hence gene expression in progeny (Figure 1), including genome-associated mechanisms such as DNA methylation as well as cytoplasmic cellular components and non-DNA-bound factors such as hormones (while ecological and cultural inheritance could possibly be included, we limit our scope to molecular systems). Although the precise regulatory impact of specific factors is not always known (and may be highly context dependent), the term IGR provides a mechanistically grounded framework to focus on heritable factors that alter gene expression and hence may be of ecological and evolutionary relevance (see Box 2 and Figure S1 in the supplemental information online for modeling implications).

Feature One: 'Non-genetic' and Genetic Aspects of Inheritance Are Inseparable 'Non-genetic' and genetic aspects of inheritance are often treated as separate streams of information, both conceptually and experimentally. Accordingly, statistical and modeling approaches
${ }^{6}$ Groningen Institute for Evolutionary Life Sciences, University of Groningen, Nijenborgh 7, 9747, AG, Groningen, The Netherlands

\section{*Correspondence:}

irene.adrian-kalchhauser@vetsuisse. unibe.ch (I. Adrian-Kalchhauser).

${ }^{\circledR}$ Twitter: @kalchhauser

(l. Adrian-Kalchhauser) and @fiwibern (Centre for Fish and Wildlife Health)

\footnotetext{
Box 1. Inherited Gene Regulation

Currently used terms such as 'epigenetic', 'non-genetic', and 'extra-genetic' bear a multiplicity of meanings in the literature (see Box 2, Figure 2 and Supplemental Table S1 in the supplemental information online for a detailed analysis of terms, their use, and their implications). Yet, the diverse molecular mechanisms involved in 'non-genetic' modes of inheritance share a common effect: they alter aspects of genome activity and affect progeny gene expression. To emphasize this essential property, Day and Bonduriansky [7] introduced the concept of 'inheritance of the gene interpretation machinery', which we modify as the more concise 'inherited gene regulation (IGR)'. Here, 'regulation' may be functional or adaptive, but (as exemplified by negative effects of 'non-genetic' inheritance) this is not necessarily the case. IGR accommodates genome-associated mechanisms as well as non-nuclear factors such as maternally provided RNA [63], proteins, hormones, or nutrients (all of which can be potent gene expression regulators [60]), and encompasses the broad concept of transgenerational plasticity $[20,33]$. Contextual specification through prefixes (e.g., gamete-mediated IGR, DNA methylation-mediated IGR, hormone-mediated IGR, or RNA-mediated IGR) permits descriptive precision while maintaining conceptual unity.
} 


\section{Inherited Gene Regulation (IGR)}
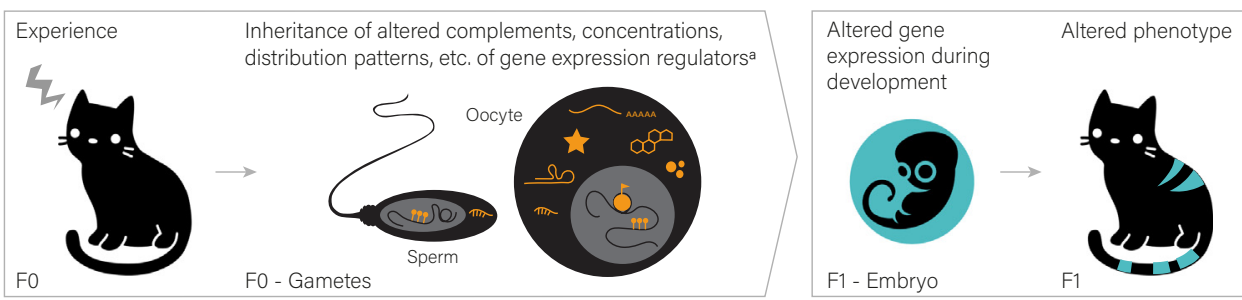

${ }^{\text {a } F o r ~ e x a m p l e . ~ t r a n s c r i p t i o n ~ f a c t o r s ~}{ }^{2}$, modified histones

noncoding RNAs $\Omega$, DNA methylation 9 i9, hormones $00^{\circ}$

nutrients and metabolites $\bullet:$, mRNAs $\sim$ anaA that encode gene

expression regulators

Trends in Ecology \& Evolution

Figure 1. Inherited Gene Regulation (IGR). IGR encompasses all inherited factors that modify gene expression in offspring. This includes a wide range of molecular pathways, molecule types, and cellular compartments. ${ }^{\star} T$ The use of cats is based on a conference remark comparing the 'dual' state of Schrödinger's cat with the dual nature of genetic/ 'non-genetic' inheritance.

often rely on the (linear) decomposition into 'genetic' and 'non-genetic' effects, and on the independent quantification of these effects (Box 2). Yet, gene sequence variants and heritable factors that regulate their activity are, in fact, deeply interwoven, suggesting that an interaction-based perspective may be more fruitful.

For reasons summarized in Figure 3A, IGR is never fully independent of DNA sequence. In the well-studied example of DNA methylation, marks are set, recognized, maintained, and erased by a large array of proteins that are encoded in the genome. Allelic variants of these genes can affect epigenetic induction and reversal dynamics [64], such that genetically distinct lines of the same species show different induced effects of parental conditions on offspring phenotype (e.g., [65-70]). In addition, DNA sequence at epigenetically targeted loci plays a role: whether or not a methylation mark can be set depends (among other factors) on the presence of CpG dinucleotides, and histone-modifying enzymes are frequently recruited by transcription factors that recognize specific DNA sequence motifs [71]. Finally, IGR mechanisms depend on the functional properties of the sequence surrounding the target locus [72]. In mammals, for instance, DNA methylation embedded in CpG-dense promoters contributes to gene silencing, whereas

\section{Term use by field}
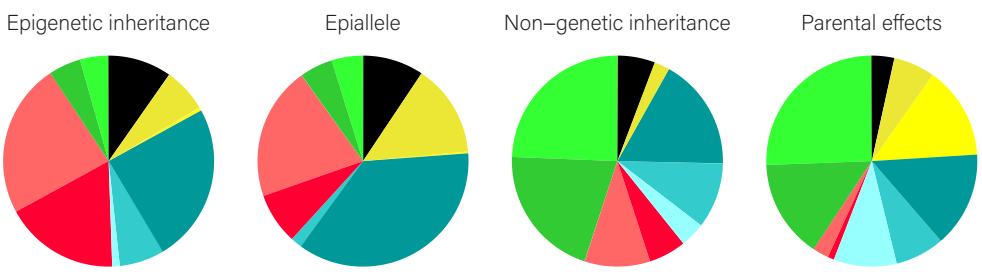

Transgenerational plasticity

$$
\begin{aligned}
& \text { Ecology } \\
& \text { Evolutionary biology } \\
& \text { Biochemistry/molecular biology } \\
& \text { Cell biology }
\end{aligned}
$$

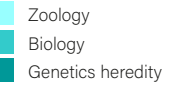

Genetics heredity

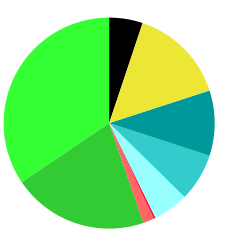

Agriculture/dairy animal science

Plant sciences

Multidisciplinary sciences

Figure 2. Terminology for 'Non-genetic' Inheritance. A search in the Web of Science reveals that the use of terminology differs by field. Raw data and methods, as well as a detailed analysis of properties, questions, and challenges associated with these and other relevant terms, are provided in Table S1 in the supplemental information online. 
Box 2. Modeling the Impact of 'Non-genetic' Inheritance

Mathematical and computational models have played a crucial role in mapping the broader implications of epigenetic modifications, maternal effects and other aspects of non-genetic inheritance (NGI) since the first models (e.g., [55]) revealed their impact on selective dynamics and outcomes. Although the two predominant modeling approaches have provided important insights, their scope is limited since current models either focus on atypical forms of $\mathrm{NGI}$ (such as epialleles at a single locus) or neglect the close interdependence of genetic and non-genetic factors (Features 1 and 3 in the main text).

In the first approach, population genetic (PG) and quantitative genetic (QG) models are expanded by including NGI. PG models explicitly include the mechanisms of genetic and NGl; even with simple scenarios (such as epialleles at a single locus, e.g., $[140,141])$, the resulting models are highly complex and difficult to analyze. To avoid these complexities, some PG models do not model inheritance directly, but instead focus on the fitness landscape [142], making the assumption that selection will shift the population to a fitness peak. In principle, fitness landscape models are versatile and can be applied to various NGI mechanisms [4]. However, current models may make unrealistic assumptions about the fitness landscape (e.g., that fitness can be split into separate genetic and epigenetic components). Moreover, when inheritance is complex, fitness may not increase during selection [143]. QG models (e.g., [55,144,145]) are technically more tractable than PG models, but they are based on strong and empirically untested assumptions such as a normal distribution of genetic and non-genetic effects, with stable variances and covariances. In particular, QG models tend to assume that genetic and non-genetic effects are additive (and therefore independent of each other) or that selection is very weak (implying that nonadditive effects are negligible). Such additivity is not supported by the available data (e.g., [146]) and does not align with an inclusive understanding of NGI as inherited gene regulatory information. By means of a Price equation approach, PG and QG models can be viewed from a unified perspective [7]. This provides useful insights, for instance, that NGI can foster rapid adaptation when a population is far from a fitness peak, while it will often lead to a fitness reduction in an already well-adapted population [56]. To date, however, applications of the Price equation (e.g., [7]) have also relied on potentially misleading simplifying assumptions such as additivity of genetic and non-genetic effects.

The second approach includes models that conceptualize the interplay of 'information channels'. Here, genetic and nongenetic factors are viewed as cues providing potentially adaptive information about the state of the environment (e.g., [5,147]). These models seek to ask what kinds of cues (i.e., inherited parental effects versus an individual's current information) will evolve to be used in a given scenario, depending on such factors as temporal versus spatial environmental fluctuations and transgenerational correlation. In contrast to most PG and QG models, the information channel approach explicitly models the machinery integrating and interpreting different kinds of information. This allows an important additional question to be addressed: how do these information-integrating systems themselves evolve? At present, however, information channel models make similar simplifying assumptions as other current models: the information-processing machinery is represented by weighing factors, and the phenotype results from the weighted summation of genetic and non-genetic cues.

To our knowledge, a mechanistic model for the evolutionary causes and consequences of NGI that addresses the three key features of these systems (see main text) has not yet been proposed. In Supplemental Figure S1, we sketch a gene regulatory network model consistent with an inclusive IGR perspective that explicitly incorporates nonadditive interactions and feedbacks among genetic, epigenetic, and environmental factors.

methylation marks embedded in coding sequences are associated with the timing of transcription initiation events, and marks in intergenic regions have little impact on genome activity [73].

At the same time, the molecular mechanisms underlying IGR feature elements of sequence independence - for example, through environmentally determined changes in the activity of pathway components, or through 'read-write' mechanisms which recognize a modification and reiterate or amplify it [74,75]. Such mechanisms account for pathway-specific fidelity properties [62]; they allow epigenetic marks to spread along chromosomes [76], to be copied to the new DNA strand during cell division [77], and to mediate the prolonged inheritance of environmental signals $[78,79]$. Overall, the molecular mechanisms underlying IGR operate on a continuum from entirely sequence-determined to completely sequence-independent (also $[53,56])$. Importantly, the location on this continuum is not fixed for any mechanism, nor for any gene. Genetic versus regulatory aspects may gain and lose importance depending on genomic location, developmental timepoint, tissue, and environmental conditions. 
(A) Non-genetic and genetic aspects of inheritance are inseparably intertwined

Interplay of genetic and non-genetic aspects in DNA methylation

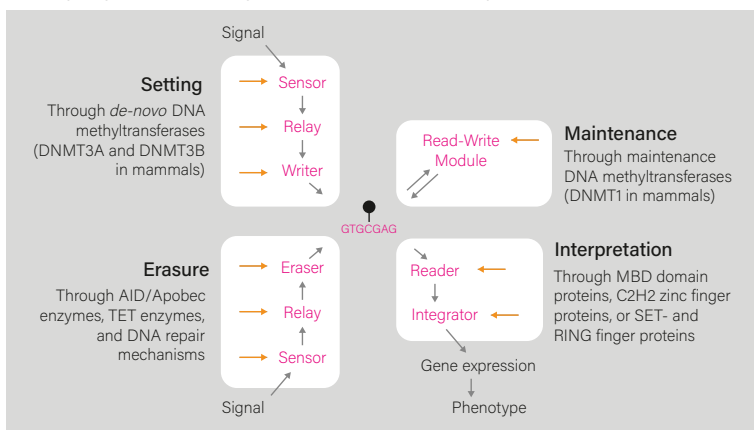

Genetic aspects

The proteins setting, maintaining, erasing, and interpreting the mark are genetically encoded. Also, the base composition and location of the target site matters.

The environment impacts the activity of genes and gene products (RNAs,

proteins) involved in setting, maintaining, erasing, and interpreting the mark.

(B) IGR mechanisms are phylogenetically and functionally diverse

Phylogenetic diversity of genetic substrates of DNA methylation and life history traits.

Three levels of diversity

A mammal and a fish are colored for comparison.

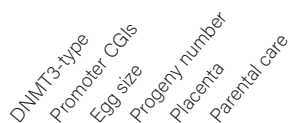

1

ic diversity

Example: within animals, clades feature from 1 to

$>10$ de-novo DNA methyltransferases, and

also differ in core traits related to IGR such

as parental care or genome architecture.

2

Dynamics diversity Example: the timing of events of DNA de- and remethylation during gametogenesis, fertilization, and embryonic developmen differs between vertebrate species

3 Example: DNA methylation has different roles in regulatory, repetitive, and intergenic

regions in the same genome.
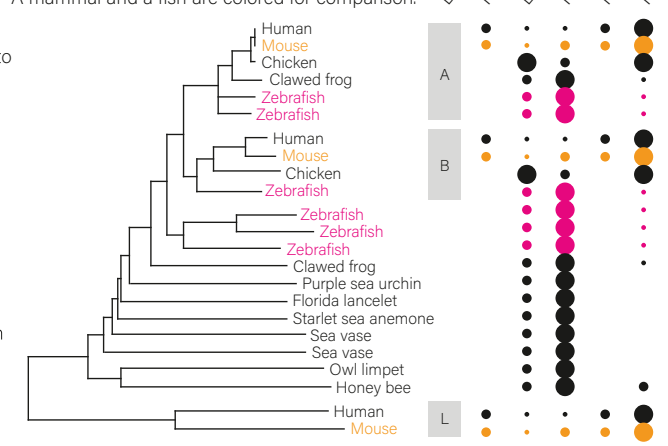

(C) IGR mechanisms are probabilistic, interactive, and context-dependent

An interactive network of mechanisms cogenerates a gene expression profile and, ultimately, a phenotype

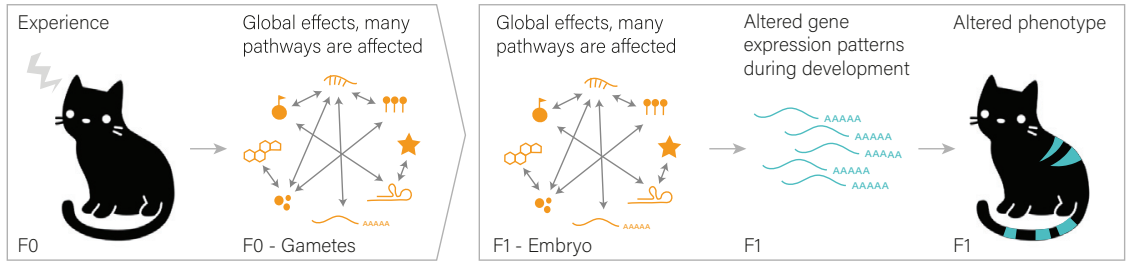

Information relay-races between mechanisms maintain information through reprogramming events
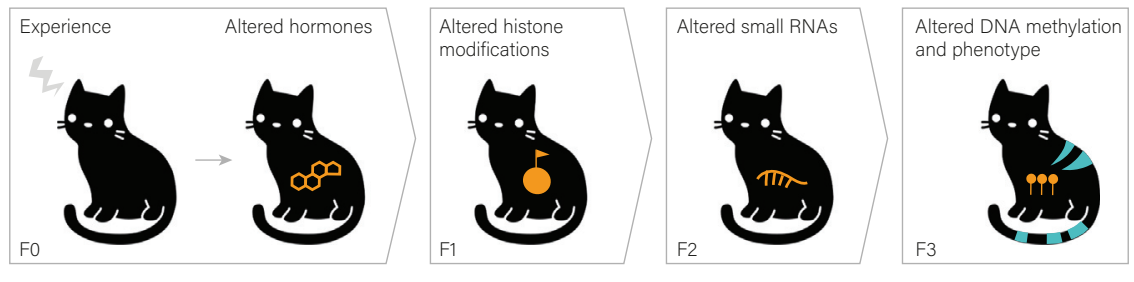

Trends in Ecology \& Evolution

Figure 3. Fundamental Features of Inherited Gene Regulation (IGR). (A) In IGR, 'non-genetic' and genetic aspects are inseparably intertwined. The pathways setting, maintaining, erasing, and interpreting a particular mark receive input from genetic and regulatory (including environmental induction) sources (shown here for DNA methylation, but the concept applies equally to other mechanisms). (B) IGR mechanisms are phylogenetically and functionally diverse. This diversity stems from multiple levels: (i) the phylogenetic level (different species feature different gene numbers and types, and

(Figure legend continued at the bottom of the next page.) 
The complex interplay of heritable regulatory factors and DNA sequence information poses considerable conceptual and experimental challenges. To face these challenges, we offer four recommendations. First, assume from the start that genetic and 'non-genetic' factors both contribute to any inherited phenomenon, and only drop this assumption in the case of unequivocal evidence to the contrary. IGR genes act pleiotropically, and even a single undetected base pair change (for example, in a master regulatory gene for DNA methylation) can potentially alter the epigenetic landscape in strains believed to be isogenic. If complete genome sequences are unavailable, and/or the effect of sequence differences is not investigated through crosses or transgenics, the relative impact of genetic and 'non-genetic' effects cannot be precisely quantified.

Second, explore how genetic variation affects IGR in your particular experimental model. Genotype or strain-specific capacities for IGR exist (see also Feature Two, later) but are poorly characterized, yet identifying loci that affect IGR capacity in natural populations could greatly increase our understanding of IGR in evolution and potentially in human disease. Third, consider a finegrained approach to the genome when assessing epigenetic and genetic variance by separately analyzing functional units such as exons, introns, promoters, enhancers, repetitive elements, and intergenic regions, or by focusing on genome regions of known phenotypic effect. A basic functional genome annotation can be created through methods such as RNA expression time courses, ATAC-seq (assay for transposase-accessible chromatin using sequencing) analyses [80], Hi-C approaches [81] or histone profile mapping [82] (also [83,84]). Technological advances bring such annotation within reach even for non-model species. A fourth alternative is to 'zoom out' rather than to 'zoom in' on the molecular picture: it may not be necessary to distinguish heritable regulatory factors from sequence information as distinct aspects of gene expression regulation, and an integrative, systems biology approach might more accurately capture a biological reality. In the theoretical domain, evolutionary models for gene-regulatory networks can be designed that explicitly address the interaction of these two components (Box 2 and Figure S1 for a sketch of such a model). Experimentally, this integration can be achieved by replacing approaches focused solely on one aspect of gene regulation, such as DNA methylation profiling, with broader measures of genome activity such as gene expression (RNA-seq), chromosomeconformation analyses (Hi-C), or overall chromatin accessibility (ATAC-seq; see references earlier). These techniques have been greatly optimized in terms of sensitivity, sample requirements and technical difficulty, making them accessible for the first time to non-experts in molecular research.

\section{Feature Two: IGR Mechanisms Are Phylogenetically and Functionally Diverse}

The mechanisms underlying IGR are evolutionarily ancient, and it is tempting to treat them as if they were as universal as the principles underlying DNA-based inheritance. However, this may be misleading, as NGI mechanisms are highly diverse. For instance, both models and experiments often assume that DNA methylation is a singular mechanism, with a uniform mode of action across the genome and in different taxa, even though differences between mammals, reptiles, and insects had been recognized by the 1980s [85]. Recognizing and deliberately

different life history traits related to reproduction and inheritance), (ii) from the level of dynamics (the same pathway may display different dynamics across fertilization in two species), and (iii) from the functional level (the same mark may have different functions in the same species, depending on the genomic context). A phylogenetic tree of de novo DNA methyltransferase genes (modified from [148]) illustrates this diversity: two of the best-studied animal models, mouse and human, feature DNMT3L genes which are absent from non-mammals. Similarly, the assignment of de-novo methyltransferases from many animals to the two 'canonical' types A and B is not straight-forward [149]. (C) IGR mechanisms are probabilistic, interactive, and context dependent. In IGR, molecular mechanisms form a 'gene-regulatory landscape' that culminates in a particular gene expression pattern and phenotype. Regulatory information can be preserved across time and generations in the absence of stable mark inheritance due to reprogramming. For a legend of symbols, see Figure 1. Abbreviations: AID, activation-induced cytidine deaminase; CGI, CpG island; DNMT, DNA methyltransferase. 
incorporating the diversity of these mechanisms into research designs can lead to important progress in understanding eco-evolutionary implications.

Diversity in IGR mechanisms is relevant at three levels (Figure 3B). First, although certain features may be broadly conserved, there is striking phylogenetic diversification in the molecular machinery for setting, maintaining, reading, and erasing epigenetic marks. For example, mammalian genomes feature three unique DNA methyltransferase DNMT genes, but teleost fishes have between five and 12 such genes ([86-90]; [91-93] for other eukaryotic examples). Phylogenetic variation has also been described for chromatin modifiers [94], histones and their variants $[95,96]$, and chromatin modification readers [97]. Second, even if different taxa feature roughly similar gene complements, the function of certain genes and the temporal or spatial dynamics of potential epigenetic modifications may differ. Such differences are particularly apparent in soma-germ line transitions in vertebrates, where the reprogramming dynamics of epigenetic setting and erasure are intensively studied. For example, modified histones are largely removed during mouse (Mus musculus) spermatogenesis but retained to some extent in human (Homo sapiens) sperm [98]. Similarly, parental DNA methylation is erased during mammalian germ cell development, but paternal methylation is largely retained in zebrafish (Danio rerio) [99,100], although less so in other fish species [101,102]. And while all taxa feature maternal RNA, clearance and maintenance dynamics differ widely [63,103-106]. A third relevant level of diversity pertains to the functional consequences of epigenetic marks, since biochemically identical marks can have very different 'meanings' in different taxa. This can be inferred at the among-species level from pattern comparisons: DNA methylation marks are concentrated within actively expressed genes in teleost fish, but are found upstream of inactive genes in mammals [107,108]. Within a species, the genomic location of a given type of mark affects its impact: functional assays indicate that DNA methylation upstream of genes in many cases inhibits gene expression, while methylation that occurs within active genes can affect the choice of transcription initiation site [109].

In summary, while most features of DNA inheritance are exceedingly highly conserved, NGl is a language with many dialects. Caution is therefore warranted when extending insights from model organisms to other taxa or strains with different evolutionary histories. This point leads to several recommendations. First, whenever possible, generalizations from distantly related species should be replaced by known properties of the species - or at least the clade - of interest. This entails addressing several questions. What is the species' phylogenetically based capacity for IGR - does the species or its clade feature RNA silencing pathways, how many DNA (de)methylases are there, does the genome feature the CpG islands in promoter regions that are most prone to methylation? What are the temporal and spatial dynamics of the mechanism of interest - are histones retained in sperm, are hormones deposited in the egg cell? Is the mechanism of interest known to be linked to gene expression in this species? Even when the answers are not all known, posing these questions can be a first step to more focused interpretation and to guide further research steps.

A related recommendation is to use a phylogenetic perspective in choosing specific research techniques. For example, in a species (such as the nematode Caenorhabditis elegans) that features dozens of Argonaute RNA-silencing genes, targeting small RNAs may be promising, but RNA isolation protocols must be fine-tuned to account for diverse small RNA types. In fish, maternal RNA is of interest, but it is important to be aware of the absence of poly-A tails on maternal messages because standard sequencing protocols rely on their presence. Affinity-based techniques to measure DNA methylation [such as MeDIP (methylated DNA immunoprecipitation) or methyl-CpG-binding domain (MBD) capture] do not perform well on loosely interspersed DNA methylation and should be avoided in species such as fish or invertebrates [110] that lack typical 
CpG islands [111-113]. We also encourage biomolecular researchers interested in IGR to incorporate evolutionary and ecological considerations into their research design. This entails using IGR triggers that are relevant to a species' naturally occurring conditions and selective forces (such as treatments that impose resource limits or biotic stresses like competition and pathogens), or choosing taxa that have evolved in response to known environmental changes $[114,115]$. These research criteria may seem obvious to the evo-ecologist but are somewhat undervalued in the molecular field.

\section{Feature Three: IGR Mechanisms Are Probabilistic, Interactive, and Context Dependent}

Views of $\mathrm{NGI}$ - and consequently, experiments and theoretical models - are largely shaped by classic cases such as paramutation of the Kit tail color gene in mice [116], flower morphology in toadflax (Linaria vulgaris) [117], or mammalian imprinting [118]. These examples are used as research models because they are robustly heritable, deterministic, and 'allelic'. However, these cases may be quite exceptional [119]. The molecular mechanisms involved in IGR most often act in a probabilistic, facultative, and context-dependent manner. Accounting for these nondeterministic properties is an important step in advancing understanding.

In what ways are IGR mechanisms nonlinear, probabilistic and context dependent? First, IGR mechanisms do not operate at the level of nucleotide resolution, but rather they integrate across larger genomic regions. In the example of a CpG-rich mammalian promoter, the combined overall state of several hundred base pairs is related to gene activity (e.g., [120]), but the methylation state of any particular nucleotide in the promoter is usually irrelevant. Treating individual occurrences of epigenetic marks like nucleotide polymorphisms (i.e., as epialleles), and analyzing 'epimutations' rather than the overall state of a genome region poorly captures the biological process [120], and might detect statistically significant but functionally uninformative differences. Second, IGR integrates information across several distinct mechanisms that spatially and temporally co-occur, work in concert, and influence each other [121-129]. This complex 'chromatin landscape' creates a gene expression profile that could not have been generated by any one mechanism alone (Figure 3C). An example is 'poised' promoters, which feature the paradoxical combination of activating and repressing histone marks on the same nucleosome [130]. Third, the stability of information is not necessarily linked to the stability of any individual mark. For example, in mice, a protein binding to previously methylated regions preserves the 'memory' of DNA methylation through meiotic demethylation in the absence of the mark itself [131-134]. Similarly, feedback loops between small RNAs and histone marks maintain information in fission yeast (Schizosaccharomyces pombe) in a phenotypically neutral state, allowing later generations to benefit from the information when required [135]. Other examples of such 'information relay races', where information about (grand)parental conditions persists in the absence of robust mark inheritance, include paternal heat exposure in wild guinea pigs (Cavia aperea) through nonconstant DNA methylation patterns [136] and offspring pathogen avoidance mediated through sequential neuronal signaling and small RNAs in C. elegans [137], see also [138]. Hence, robust inheritance of any single mark across more than one generation is not necessarily required for multigenerational effects on the phenotype (Figure 3C).

In sum, IGR mechanisms act as a cluster of interdependent molecular nudges that together enhance the likelihood of a particular outcome on the gene expression level. This complexity creates challenges for empirical studies, which often aim to track down the effect of a single type of mechanism or a single localized change. How can we address this challenge in designing ecoevolutionary studies of NGI? One approach is to design experiments to investigate the 'cluster of nudges' in its entirety, for example, by starting investigations at the integrative level of gene 
expression (e.g., with RNA-seq approaches), or by investigating multiple pathways (DNA methylation, small RNAs, histone modifications, nutrients, metabolites, hormones) in parallel. Key decisions must also be made in assessing the outcome. IGR may manifest in developing offspring only under certain conditions [29], and it may be useful to measure gene expression at early life-cycle points (e.g., embryos or even gametes) in which developmental responses to laboratory conditions may be less likely to overwrite any inherited alterations [139]. Functional analyses of early-life gene expression can also help guide the choice of adult traits to study. For example, differential expression analyses of the offspring of drought-stressed plants might reveal down-regulation of small RNAs targeting root-related processes, suggesting a focus on root extension and uptake rates rather than on leaf traits or transition to flowering. Importantly, tissue-specific approaches to gene expression analyses should always be preferred (even if they require delicate dissections) to avoid losing signals that manifest only in particular tissues.

\section{Concluding Remarks}

A key contemporary research challenge is incorporating inheritance mechanisms beyond DNA sequence into evolutionary and ecological investigations. Some simplification of these dauntingly diverse and functionally complex mechanisms is reasonable and indeed necessary for this effort. By drawing on molecular insights to these mechanisms, this can be done in ways that maintain rather than distort key aspects of their functionality. 'Cross-talk' between evolutionary and molecular biologists provides a way to bridge this gap in understanding. Recognizing the common effect of highly diverse molecular mechanisms as IGR is a first step toward identifying general features of NGI systems. Characterizing such features can help replace some initial misconceptions with a more solid mechanistic foundation to inform ecology and evolution theory and research programs. IGR seems a quintessential area where collaboration between molecular specialists and those with knowledge of ecological and evolutionary issues can be especially productive on both sides (see also Outstanding Questions).

\section{Acknowledgments}

The idea for this paper was initially proposed by I.A.-K. and was further developed by all authors in a workshop generously funded by grant No 789240 from the European Research Council (ERC) to F.J.W. S.E.S. acknowledges support from Wesleyan University and The John Templeton Foundation. The authors thank the Editor and two anonymous reviewers for their constructive comments on an earlier version of the manuscript.

\section{Supplemental Information}

Supplemental information associated with this article can be found online at https://doi.org/10.1016/j.tree.2020.08.011.

\section{References}

1. Klosin, A. and Lehner, B. (2016) Mechanisms, timescales and principles of trans-generational epigenetic inheritance in animals. Curr. Opin. Genet. Dev. 36, 41-49

2. Grossniklaus, U. et al. (2013) Transgenerational epigenetic inheritance: how important is it? Nat. Rev. Genet. 14, 228-235

3. Danchin, É. et al. (2011) Beyond DNA: integrating inclusive inheritance into an extended theory of evolution. Nat. Rev. Genet. 12, 475-486

4. Danchin, É et al. (2019) Epigenetically facilitated mutational assimilation: epigenetics as a hub within the inclusive evolutionary synthesis. Biol. Rev. Camb. Philos. Soc. 94, 259-282

5. McNamara, J.M. et al. (2016) Detection vs. selection: integration of genetic, epigenetic and environmental cues in fluctuating environments. Ecol. Lett. 19, 1267-1276

6. Verhoeven, K.J.F. and Preite, V. (2014) Epigenetic variation in asexually reproducing organisms. Evolution 68, 644-655

7. Day, T. and Bonduriansky, R. (2011) A unified approach to the evolutionary consequences of genetic and nongenetic inheritance. Am. Nat. 178, 18-36
8. Bonduriansky, R. et al. (2012) The implications of nongenetic inheritance for evolution in changing environments. Evol. Appl. 5, 192-201

9. Sultan, S.E. (2015) Organism and Environment. Ecological Develop-

10. Jablonka, E. and Raz, G. (2009) Transgenerational epigenetic inheritance: prevalence, mechanisms, and implications for the study of heredity and Evolution. Q. Rev. Biol. 84, 131-176

11. Blake, G.E.T. and Watson, E.D. (2016) Unravelling the complex mechanisms of transgenerational epigenetic inheritance. Curr. Opin. Chem. Biol. 33, 101-107

12. Perez, M.F. and Lehner, B. (2019) Intergenerational and transgenerational epigenetic inheritance in animals. Nat. Cell Biol. 21, 143-151

13. Quadrana, L. and Colot, V. (2016) Plant transgenerational epigenetics. Annu. Rev. Genet. 50, 467-491

14. Paszkowski, J. and Grossniklaus, U. (2011) Selected aspects of transgenerational epigenetic inheritance and resetting in plants. Curr. Opin. Plant Biol. 14, 195-203 ment, Niche Construction, and Adaptation, Oxford University Press

\section{Outstanding Questions}

What are the key functional features of inheritance systems beyond DNA sequence with regard to adaptive variation and evolution? Studies of induction and reversal cues and dynamics, phenotypic impact, and potential cumulative effects across generations are of particular relevance.

Given the interactive and reciprocating functionality of genetic and 'nongenetic' factors, how can these aspects of inheritance most productively be addressed? Treating them as separate elements may be less informative for understanding gene regulatory effects than an integrated approach, which will require changes to experimental design.

How can the interplay of genetic and 'non-genetic' factors be incorporated in eco-evolutionary models in a realistic manner? The close interaction of genetic and 'non-genetic' regulatory elements calls for the development of regulatory network models, together with new tools for deriving overarching principles from such mechanistic models.

How do specific IGR mechanisms vary in different phylogenetic, environmental, and tissue contexts? Accounting for this diversity and complexity calls for expanding empirical studies far beyond the most well-known model organisms and standard laboratory conditions. 
15. Johannes, F. et al. (2009) Assessing the impact of transgenerational epigenetic variation on complex traits. PLoS Genet. 5, e1000530

16. Laland, K. et al. (2014) Does evolutionary theory need a rethink? Nature 514, 161-164

17. Charlesworth, D. et al. (2017) The sources of adaptive variation. Proc. R. Soc. Lond. B Biol. Sci. 284, 20162864

18. Uller, T. et al. (2013) Weak evidence for anticipatory parental effects in plants and animals. J. Evol. Biol. 26, 2161-2170

19. Salinas, S. et al. (2013) Non-genetic inheritance and changing environments. Non-Gen. Inh. 1, 38-50

20. Herman, J. and Sultan, S. (2011) Adaptive transgenerational plasticity in plants: case studies, mechanisms, and implications for natural populations. Front. Plant Sci. 2, 102

21. Maestripieri, D., Mateo, J.M., eds (2009) Maternal Effects in Mammals, The University of Chicago Press

22. Badyaev, A.V. and Uller, T. (2009) Parental effects in ecology and evolution: mechanisms, processes and implications. Philos. Trans. R. Soc. Lond. B Biol. Sci. 364, 1169-1177

23. Öst, A. et al. (2014) Paternal diet defines offspring chromatin state and intergenerational obesity. Cell 159, 1352-1364

24. Chen, Q. et al. (2016) Sperm tsRNAs contribute to intergenertional inheritance of an acquired metabolic disorder Science 351, 397-400

25. Gluckman, P.D. et al. (2009) Epigenetic mechanisms that underpin metabolic and cardiovascular diseases. Nat. Rev. Endocrinol. 5, 401-408

26. Shama, L.N.S. et al. (2014) Transgenerational plasticity in ma rine sticklebacks: maternal effects mediate impacts of a warming ocean. Funct. Ecol. 28, 1482-1493

27. Schunter, C. et al. (2018) An interplay between plasticity and parental phenotype determines impacts of ocean acidification on a reef fish. Nat. Ecol. Evol. 2, 334-342

28. Ryu, T. et al. (2018) The epigenetic landscape of transgenerational acclimation to ocean warming. Nat. Clim. Chang. 8, 504-509

29. Baker, B.H. et al. (2018) Context-dependent developmenta effects of parental shade versus sun are mediated by DNA methylation. Front. Plant Sci. 9, 125

30. Scoville, A.G. et al. (2011) Differential regulation of a MYB transcription factor is correlated with transgenerational epigenetic inheritance of trichome density in Mimulus guttatus. New Phytol. 191, 251-263

31. Herman, J.J. et al. (2012) Adaptive transgenerational plasticity in an annual plant: grandparental and parental drought stress enhance performance of seedlings in dry soil. Integr. Comp. Biol. 52, 77-88

32. Baker, B.H. et al. (2019) Transgenerational effects of parenta light environment on progeny competitive performance and lifetime fitness. Philos. Trans. R. Soc. Lond. B Biol. Sci. 374 20180182

33. Donelson, J.M. et al. (2018) Transgenerational plasticity and climate change experiments. Where do we go from here? Glob. Chang. Biol. 24, 13-34

34. Fox, R.J. et al. (2019) Beyond buying time: the role of plasticity in phenotypic adaptation to rapid environmental change. Philos. Trans. R. Soc. Lond. B Biol. Sci. 374, 20180174

35. Hofmann, G.E. (2017) Ecological epigenetics in marine metazoans. Front. Mar. Sci. Published online January 18 2017. https://doi.org/10.3389/fmars.2017.00004

36. Flores, K.B. et al. (2013) The role of methylation of DNA in en vironmental adaptation. Integr. Comp. Biol. 53, 359-372

37. Richards, C.L. et al. (2012) Evolutionary significance of epigenetic variation. In Plant Genome Diversity Volume 1 Wendel, J.F. et al., eds), pp. 257-274, Springer, Vienna

38. Meröndun, J. et al. (2019) Genome-scale sampling suggests cryptic epigenetic structuring and insular divergence in Canada lynx. Mol. Ecol. 28, 3186-3196

39. Zhang, Y.-Y. et al. (2018) Understanding the evolutionary potential of epigenetic variation: a comparison of heritable phenotypic variation in epiRILs, RILs, and natural ecotypes of Arabidopsis thaliana. Heredity (Edinb.) 121, 257-265

40. Rey, O. et al. (2020) Linking epigenetics and biological conservation: towards a conservation epigenetics perspective. Funct. Ecol. 34, 414-427
41. Hu, J. and Barrett, R.D.H. (2017) Epigenetics in natural animal populations. J. Evol. Biol. 30, 1612-1632

42. Baldanzi, S. et al. (2017) Epigenetic variation among natura populations of the South African sandhopper Talorchestia capensis. Evol. Ecol. 31, 77-91

43. Marin, P. et al. (2020) Biological invasion: the influence of the hidden side of the (epi)genome. Funct. Ecol. 34, 385-400

44. Ardura, A. et al. (2017) Epigenetic signatures of invasive status in populations of marine invertebrates. Sci. Rep. 7, 42193

45. Baerwald, M.R. et al. (2016) Migration-related phenotypic divergence is associated with epigenetic modifications in rainbow trout. Mol. Ecol. 25, 1785-1800

46. Kesäniemi, J.E. et al. (2016) DNA methylation and potential fo epigenetic regulation in Pygospio elegans. PLOS ONE 11, e0151863

47. Kucharski, R et al. (2008) Nutritional control of reproductive status in honeybees via DNA methylation. Science 319 , 1827-1830

48. Hu, J. et al. (2018) Genome-wide DNA methylation signatures of infection status in Trinidadian guppies (Poecilia reticulata). Mol. Ecol. 27, 3087-3102

49. Keller, T.E. et al. (2016) The multivariate association between genomewide DNA methylation and climate across the range of Arabidopsis thaliana. Mol. Ecol. 25, 1823-1837

50. Palumbi, S.R. et al. (2014) Mechanisms of reef coral resistance to future climate change. Science 344, 895-898

51. Heckwolf, M.J. et al. (2020) Two different epigenetic information channels in wild three-spined sticklebacks are involved in salinity adaptation. Sci. Adv. 6, eaaz1138

52. Richards, C.L. et al. (2017) Ecological plant epigenetics. Evidence from model and non-model species, and the way forward. Ecol. Lett. 20, 1576-1590

53. Richards, E.J. (2006) Inherited epigenetic variation - revisiting soft inheritance. Nat. Rev. Genet. 7, 395-401

54. Uller, T. (2019) Chapter 15 - Evolutionary perspectives on transgenerational epigenetics. In Transgenerational Epigenetics (Second Edition): Translational Epigenetics (Tollefsbol, T.O., ed.), pp. 333-350, Academic Press

55. Kirkpatrick, M. and Lande, R. (1989) The evolution of materna characters. Evolution 43, 485-503

56. Bonduriansky, R. and Day, T. (2018) Extended Heredity: A New Understanding of Inheritance and Evolution, Princeton University Press

57. Eirin-Lopez, J.M and Putnam, H.M. (2019) Marine environmental epigenetics. Annu. Rev. Mar. Sci. 11, 335-368

58. Skvortsova, K. et al. (2018) Functions and mechanisms of epigenetic inheritance in animals. Nat. Rev. Mol. Cell Biol. 19, 774-790

59. Miska, E.A. and Ferguson-Smith, A.C. (2016) Transgenerational inheritance: models and mechanisms of non-DNA sequence-based inheritance. Science 354, 59-63

60. Wong, C.C. et al. (2017) Interplay between epigenetics and metabolism in oncogenesis: mechanisms and therapeutic approaches. Oncogene 36, 3359-3374

61. Niederhuth, C.E. et al. (2016) Widespread natural variation of DNA methylation within angiosperms. Genome Biol. 17, 194

62. van der Graaf, A. et al. (2015) Rate, spectrum, and evolutionary dynamics of spontaneous epimutations. Proc. Natl. Acad. Sci. U. S. A. 112, 6676-6681

63. Tadros, W. and Lipshitz, H.D. (2009) The maternal-to-zygotic transition: a play in two acts. Development 136, 3033-3042

64. Gutierrez-Arcelus, M. et al. (2013) Passive and active DNA methylation and the interplay with genetic variation in gene regulation. eLife 2, e00523

65. Valdivieso, A. et al. (2020) Exposure of zebrafish to elevated temperature induces sex ratio shifts and alterations in the testicular epigenome of unexposed offspring. Environ. Res. 186, 109601

66. Vu, W.T. et al. (2015) Genetic variation of transgenerational plasticity of offspring germination in response to salinity stress and the seed transcriptome of Medicago truncatula. BMC Evol. Biol. 15, 59

67. Plaistow, S.J. et al. (2015) Offspring provisioning explains clone-specific maternal age effects on life history and life span in the water flea, Daphnia pulex. Am. Nat. 186, 376-389 
68. Schmitt, D.P. and Shannon, G. (1992) Differentiating soybean responses to Heterodera glycines races. Crop Sci. 32, 275-277

69. Stjernman, M. and Little, T.J. (2011) Genetic variation for maternal effects on parasite susceptibility. J. Evol. Biol. 24, 2357-2363

70. Sultan, S.E. (2019) Genotype-Environment Interaction and the Unscripted Reaction Norm. Cause and Process in Evolution, MIT Press

71. Stadler, M.B. et al. (2011) DNA-binding factors shape the mouse methylome at distal regulatory regions. Nature 480 , 490-495

72. Baubec, T. and Schübeler, D. (2014) Genomic patterns and context specific interpretation of DNA methylation. Curr. Opin. Genet. Dev. 25, 85-92

73. Greenberg, M.V.C. and Bourc'his, D. (2019) The diverse roles of DNA methylation in mammalian development and disease. Nat. Rev. Mol. Cell Biol. 20, 590-607

74. Margueron, R. et al. (2009) Role of the polycomb protein EED in the propagation of repressive histone marks. Nature 461 , 762-767

75. Ragunathan, K. et al. (2015) Epigenetics. Epigenetic inheritance uncoupled from sequence-specific recruitment. Science 348,1258699

76. Wutz, A. and Valencia, K. (2015) Recent insights into the regulation of X-chromosome inactivation. Adv. Genomics Genet. 5 , 227-238

77. Escobar, T.M. et al. (2019) Active and repressed chromatin domains exhibit distinct nucleosome segregation during DNA replication. Cell 179, 953-963

78. Rechavi, O. et al. (2014) Starvation-induced transgenerationa inheritance of small RNAs in C. elegans. Cell 158, 277-287

79. Houri-Zeevi, L. and Rechavi, O. (2017) A matter of time. Sma RNAs regulate the duration of epigenetic inheritance. Trends Genet. 33, 46-57

80. Buenrostro, J.D. et al. (2015) ATAC-seq: a method for assaying chromatin accessibility genome-wide. Curr. Protoc Mol. Biol. 109, 21.29.1-21.29.9

81. Ron, G. et al. (2017) Promoter-enhancer interactions identified from $\mathrm{Hi}-\mathrm{C}$ data using probabilistic models and hierarchical topological domains. Nat. Commun. 8, 2237

82. Schwaiger, M. et al. (2014) Evolutionary conservation of the eumetazoan gene regulatory landscape. Genome Res. 24, 639-650

83. Lu, Z. et al. (2019) The prevalence, evolution and chromatin signatures of plant regulatory elements. Nat. Plants 5 , 1250-1259

84. Villar, D. et al. (2015) Enhancer evolution across 20 mammalian species. Cell 160, 554-566

85. Bird, A.P. and Taggart, M.H. (1980) Variable patterns of tota DNA and rDNA methylation in animals. Nucleic Acids Res. 8 , 1485-1497

86. Liu, J. et al. (2020) Evolutionary history of DNA methylation related genes in chordates: new insights from multiple whole genome duplications. Sci. Rep. 10, 970

87. Campos, C. et al. (2012) Molecular evolution of zebrafish dnmt3 genes and thermal plasticity of their expression during embryonic development. Gene 500, 93-100

88. Yokomine, T. et al. (2006) Evolution of the vertebrate DNMT3 gene family: a possible link between existence of DNMT3L and genomic imprinting. Cytogenet. Genome Res. 113, 75-80

89. Ponger, L. and Li, W.-H. (2005) Evolutionary diversification of DNA methyltransferases in eukaryotic genomes. Mol. Biol. Evol. 22, 1119-1128

90. Fellous, A. and Shama, L.N.S. (2019) Genome survey of chromatin-modifying enzymes in threespine stickleback: crucial epigenetic toolkit for Adaptation? Front. Mar. Sci. Published online November 20, 2019. https://doi.org/ 10.3389/fmars.2019.00721

91. Catania, S. et al. (2020) Evolutionary persistence of DNA methylation for millions of years after ancient loss of a de novo methyltransferase. Cell 180, 263-277

92. Capuano, F. et al. (2014) Cytosine DNA methylation is found in Drosophila melanogaster but absent in Saccharomyces cerevisiae, Schizosaccharomyces pombe, and other yeast species. Anal. Chem. 86, 3697-3702

93. Alvarez-Ponce, D. et al. (2018) Molecular evolution of DNMT1 in vertebrates. Duplications in marsupials followed by positive selection. PLOS ONE 13, e0195162

94. Liu, X. et al. (2015) Evolution and coevolution of PRC2 genes in vertebrates and mammals. Adv. Protein Chem. Struct. Biol. 101, 125-148

95. Henikoff, S. and Smith, M.M. (2015) Histone variants and epigenetics. Cold Spring Harb. Perspect. Biol. 7, a019364

96. Balhorn, R. (2007) The protamine family of sperm nuclear proteins. Genome Biol. 8, 227

97. Helleu, Q. and Levine, M.T. (2018) Recurrent amplification of the heterochromatin protein 1 (HP1) gene family across Diptera. Mol. Biol. Evol. 35, 2375-2389

98. Brykczynska, U. et al. (2010) Repressive and active histone methylation mark distinct promoters in human and mouse spermatozoa. Nat. Struct. Mol. Biol. 17, 679-687

99. Skvortsova, K. et al. (2019) Retention of paternal DNA methylome in the developing zebrafish germline. Nat. Commun. 10, 3054

100. Ortega-Recalde, O. et al. (2019) Zebrafish preserve global germline DNA methylation while sex-linked rDNA is amplified and demethylated during feminisation. Nat. Commun. 10, 3053

101. Fellous, A. et al. (2018) DNA methylation in adults and during development of the self-fertilizing mangrove rivulus, Kryptolebias marmoratus. Ecol. Evol. 8, 6016-6033

102. Wang, X. and Bhandari, R.K. (2019) DNA methylation dynamics during epigenetic reprogramming of medaka embryo. Epigenetics 14, 611-622

103. Wagner, D.S. et al. (2004) Maternal control of development at the midblastula transition and beyond: mutants from the zebrafish II. Dev. Cell 6, 781-790

104. Yartseva, V. and Giraldez, A.J. (2015) The maternal-to-zygotic transition during vertebrate development. A model for reprogramming. Curr. Top. Dev. Biol. 113, 191-232

105. Abrams, E.W. and Mullins, M.C. (2009) Early zebrafish development: it's in the maternal genes. Curr. Opin. Genet. Dev. 19, 396-403

106. Jukam, D. et al. (2017) Zygotic genome activation in vertebrates. Dev. Cell 42, 316-332

107. McGaughey, D.M. et al. (2014) Genomics of CpG methylation in developing and developed zebrafish. G3 (Bethesda) 4, 861-869

108. Han, L. and Zhao, Z. (2008) Comparative analysis of CpG islands in four fish genomes. Comp. Funct. Genomics 2008, 565631

109. Teissandier, A. and Bourc'his, D. (2017) Gene body DNA methylation conspires with $\mathrm{H} 3 \mathrm{~K} 36 \mathrm{me} 3$ to preclude aberrant transcription. EMBO J. 36, 1471-1473

110. Jiang. N. et al. (2014) Conserved and divergent patterns of DNA methylation in higher vertebrates. Genome Biol. Evol. 6 , 2998-3014

111. Nair, S.S. et al. (2011) Comparison of methyl-DNA immunoprecipitation (MeDIP) and methyl-CpG binding domain (MBD) protein capture for genome-wide DNA methylation analysis revea CpG sequence coverage bias. Epigenetics 6, 34-44

112. Harris, R.A. et al. (2010) Comparison of sequencing-based methods to profile DNA methylation and identification of monoallelic epigenetic modifications. Nat. Biotechnol. 28, 1097-1105

113. Bock, C. et al. (2010) Quantitative comparison of genome-wide DNA methylation mapping technologies. Nat. Biotechnol. 28 , 1106-1114

114. Kuijper, B. and Johnstone, R.A. (2016) Parental effects and the evolution of phenotypic memory. J. Evol. Biol. 29, 265-276

115. Uller, T. (2008) Developmental plasticity and the evolution of parental effects. Trends Ecol. Evol. (Amst.) 23, 432-438

116. Rassoulzadegan, M. et al. (2006) RNA-mediated nonmendelian inheritance of an epigenetic change in the mouse. Nature 441, 469-474

117. Cubas, P. et al. (1999) An epigenetic mutation responsible for natural variation in floral symmetry. Nature 401, 157-161

118. Patten, M.M. et al. (2014) The evolution of genomic imprinting Theories, predictions and empirical tests. Heredity (Edinb.) 113, $119-128$ 
119. Kazachenka, A. et al. (2018) Identification, characterization, and heritability of murine metastable epialleles. Implications for non-genetic inheritance. Cell 175, 1259-1271

120. Weber, M. et al. (2007) Distribution, silencing potential and evolutionary impact of promoter DNA methylation in the human genome. Nat. Genet. 39, 457-466

121. Akmammedov, A. et al. (2019) Bivalency in Drosophila embryos is associated with strong inducibility of Polycomb target genes. Fly 13, 42-50

122. Muers, M. (2011) The modENCODE guide to the genome. Nat. Rev. Genet. 12, 80

123. Davis, C.A. et al. (2018) The Encyclopedia of DNA elements (ENCODE). Data portal update. Nucleic Acids Res. 46, 794-801

124. Marchal, C. and Miotto, B. (2015) Emerging concept in DNA methylation. Role of transcription factors in shaping DNA methylation patterns. J. Cell. Physiol. 230, 743-751

125. Feldmann, A. et al. (2013) Transcription factor occupancy can mediate active turnover of DNA methylation at regulatory regions. PLoS Genet. 9, e1003994

126. Dolinoy, D.C. et al. (2010) Variable histone modifications at the Avy metastable epiallele. Epigenetics 5, 637-644

127. Meissner, A. et al. (2008) Genome-scale DNA methylation maps of pluripotent and differentiated cells. Nature 454, 766-770

128. Rose, N.R. and Klose, R.J. (2014) Understanding the relationship between DNA methylation and histone lysine methylation. Biochim. Biophys. Acta Gene Regul. Mech. 1839, 1362-1372

129. Jenuwein, T. and Allis, C.D. (2001) Translating the histone code. Science 293, 1074

130. Bernstein, B.E. et al. (2006) A bivalent chromatin structure marks key developmental genes in embryonic stem cells. Cell 125, 315-326

131. Kremsky, I. and Corces, V.G. (2020) Protection from DNA remethylation by transcription factors in primordial germ cells and pre-implantation embryos can explain trans-generational epigenetic inheritance. Genome Biol. Published online May 18, 2020. https://doi.org/10.1186/s13059-020-02036-w

132. Li, X. et al. (2008) A maternal-zygotic effect gene, Zfp57, maintains both maternal and paternal imprints. Dev. Cell 15, 547-557

133. Sampath Kumar, A. et al. (2017) Loss of maternal Trim28 causes male-predominant early embryonic lethality. Genes Dev. 31, 12-17

134. Messerschmidt, D.M. (2012) Should I stay or should I go. Protection and maintenance of DNA methylation at imprinted genes. Epigenetics 7, 969-975
135. Duempelmann, L. et al. (2019) Inheritance of a phenotypically neutral epimutation evokes gene silencing in later generations. Mol. Cell 74, 534-541

136. Weyrich, A. et al. (2016) Paternal heat exposure causes DNA methylation and gene expression changes of in wild guinea pig sons. Ecol. Evol. 6, 2657-2666

137. Moore, R.S. et al. (2019) Piwi/PRG-1 Argonaute and TGF- $\beta$ mediate transgenerational learned pathogenic avoidance. Cell 177, 1827-1841

138. Burton, N.O. et al. (2017) Insulin-like signalling to the maternal germline controls progeny response to osmotic stress. Nat. Cell Biol. 19, 252-257

139. Auge, G.A. et al. (2017) Adjusting phenotypes via within- and across-generational plasticity. New Phytol. 216, 343-349

140. Geoghegan, J.L. and Spencer, H.G. (2012) Populationepigenetic models of selection. Theor. Popul. Biol. 81, 232-242

141. Geoghegan, J.L. and Spencer, H.G. (2013) The adaptive invasion of epialleles in a heterogeneous environment. Theor. Popul. Biol. 88, 1-8

142. Klironomos, F.D. et al. (2013) How epigenetic mutations can affect genetic evolution: model and mechanism. Bioessays 35, $571-578$

143. Weissing, F.J. (1996) Genetic versus phenotypic models of selection: can genetics be neglected in a long-term perspective? J. Math. Biol. 34, 533-555

144. Bonduriansky, R. and Day, T. (2008) Nongenetic inheritance and its evolutionary implications. Annu. Rev. Ecol. Evol. Syst. 40, 103-125

145. Kuijper, B. and Hoyle, R.B. (2015) When to rely on maternal effects and when on phenotypic plasticity? Evolution 69 950-968

146. Stein, L.R. et al. (2018) Personal and transgenerational cues are nonadditive at the phenotypic and molecular level. Nat. Ecol. Evol. 2, 1306-1311

147. English, S. et al. (2015) The information value of non-genetic inheritance in plants and animals. PLOS ONE 20, e0116996

148. Albalat, R. (2008) Evolution of DNA-methylation machinery: DNA methyltransferases and methyl-DNA binding proteins in the amphioxus Branchiostoma floridae. Dev. Genes Evol. 218, 691-701

149. Liu, J.Hu, H.Panserat, S. et al. (2020) Evolutionary history of DNA methylation related genes in chordates: new insights from multiple whole genome duplications. Sci. Rep. 10, 970 